\title{
Hydrophilic lenses for 'continuous' wear in aphakia: Definitive fitting and the problems that occur
}

\author{
H. JONATHAN KERSLEY, CHRISTOPHER KERR, AND DERMOT PIERSE \\ Croydon Eye Unit
}

SUMMARY Patients have been very satisfied with these lenses ( $78 \%$ wearing at the end of six months), not the least those with reduced function of the macula. The problems of infection have been discussed, and apart from improvement in lens materials patient selection is of paramount importance. The problem of deposits is presented, but their elimination will depend on more research into their nature and development, improvement of the material's resistance to this form of attack, and improved lens design to overcome corneal and tear flow problems.

This is a clinical study of 50 patients fitted with soft lenses for 'continuous' wear at approximately six weeks after operation and their follow-up for six months. The advantages of corneal contact lenses in aphakia mentioned in the first article (above) also apply broadly to soft lenses. However, previous studies with soft lenses for daily wear have shown their limitation.

The conclusions from a trial with the Bausch and Lomb Soflens for daily wear (Kersley and Gasson, 1975) were that the stock system of supply worked well in hospital practice, that the lens was fairly well tolerated even in corneal contact lens failure, but that the spin casting method of manufacture lacked the flexibility of approach to fitting that was desirable, the handling of lenses was difficult, the visual acuity less than with spectacles (an average of 0.51 lines), and the breakage rate unacceptably high (three lenses per patient for six months).

When the higher water content lens (Sauflon 70) was tried for daily wear (Kersley, 1975) the conclusions were that the lens was better tolerated, the lathe-cut lens was better for fitting and gave better vision, but the lenses were less well reproduced, were more fragile (four lenses per patient per six months), and 'custom' making of lenses resulted in delay in supply.

From these two trials it will be realised that in aphakia the lens required needs to be: of high water content, lathe cut, fitted from stock, free from handling and breakage problems.

A 'continuously' worn lens would satisfy this last requirement, and the development of Sauflon 85

Address for reprints: Mr H. Jonathan Kersley, Croydon Eye Unit, Mayday Road, Thornton Heath, Surrey CR4 7XN. seemed to provide a material that could fulfil all the requirements of the ideal lens. Chemically the material is a cross-linked copolymer in which the comonomers are polymethylmethacrylate and vinyl pyrrolidone, compared with the HEMA based materials that are cross-linked homopolymers of hydroxyethylmethacrylate (PolyHEMA) and ethylene glycol dimethacrylate. Sauflon 85 has a water uptake of $85 \%$ and oxygen permeability of 900 units. This compares with the HEMA materials whose oxygen permeability is 40 units when the unit of oxygen permeability is $\frac{\mathrm{cm} \mathrm{sec} \mathrm{cm}^{2}(\mathrm{cmHg})}{\left(\mathrm{cc} \mathrm{O}_{2} \text { STP } 10^{-10}\right)}$ (Larke and Hirji, 1974). The relationship is, however, not linear.

This, as mentioned in connection with the fitting at operation, even with the thickness of an aphakic lens allows a near physiological volume of oxygen to pass to the cornea with the lids closed. The other parameters of lenses used are the same as before (back optic radii of $7.8 \mathrm{~mm}$ and $8.1 \mathrm{~mm}$, diameter of 13.50 , reduced front optic of 8.0 , and an average centre thickness of about $0 \cdot 50$ ).

Of the 50 patients in this series 41 had been fitted at operation ( $64 \%$ of the 'fitted at operation' series), and this raised the point that a decision had to be made during the postoperative period about the most appropriate optical device for a particular patient. This will receive more discussion later.

The fitting of these lenses was carried out at about six weeks after operation. After slit lamp examination, refraction, and keratometry a final decision was made as to soft lens fitting and a lens chosen from a stock of 44 lenses. The appropriate power had been calculated from the mean ocular 
sphere and the curvature chosen as that nearest to but not steeper than the flattest $K$ reading. The lens was then inserted and left for half an hour so that the swell factor and temperature could adjust before the lens fit was assessed. At this time, as at all other assessments, particular attention was paid to centration, lens movement, over-refraction, and perilimbal vascularity. Our aim was to produce a slightly loose-fitting lens so that a 'tight' syndrome would not occur under adverse conditions.

At the end of this first visit the patient was given an explanation about what had been done and told of the necessity for attending regularly for follow-up. Lens handling being the major problem of soft lenses in aphakia, patients were not taught how to remove their lens but were all told to report immediately if redness, soreness, or reduced vision occurred.

The second visit was within 24 hours to check the absence of corneal oedema and reassess lens fit. The third visit was one week later, and thereafter patients were examined monthly. At these visits the following were recorded: visual acuity, overcorrection, lens fit, slit lamp findings.

Overcorrections were given where indicated and reading additions prescribed. At the final visit keratometry was performed, the cornea stained with fluorescein, and the lens cleaned chemically and then heat 'sterilised'. The results are shown in Table 1.

Patients were never rejected on the grounds of poor central vision as these patients would be even more hampered by the added difficulties of aphakic spectacles (as previously described) and would find the handling of other contact lenses extremely difficult. In fact reduced central vision in aphakia may in itself be an indication for a continuously worn soft lens as a low visual aid.

Rejection of patients at the outset (Table 2) centred around high astigmatism, particularly when associated with good visual acuity or an established aphakic correction in the other eye. Those taken off the trial (Table 3) contain lens stability problems for the most part, one insuperable greasing problem, one surgical problem, and three were removed for social reasons.

The choice of lens radius was reflected in the average flattest keratometry (Table 4). The increased corneal astigmatism that occurs in aphakia accounts for our bias towards an apparently steeper fitting. Four radius changes were necessary during the trial-one flatter because of a tight lens edge and three steeper because of poor centration. Comparison of the flattest keratometry at six weeks and six months (Table 4) shows a general flattening (but not enough to affect lens fit), while the change in average mean keratometry shows the constancy of this figure that we had found before.
Table 1 Results

\begin{tabular}{lr}
\hline 50 consecutive cases & $100 \%$ \\
39 completed the trial & $78 \%$ \\
8 taken off the trial & $16 \%$ \\
3 rejected at outset & $6 \%$
\end{tabular}

Of those completing the trial:

Ages 34 to 90 . Average 71 years.

Males 15 (39.5\%). Females $23(60 \cdot 5 \%)$.

Table 2 Patients rejected from the trial ( 3 cases)

Peripheral corneal degeneration with vascularisation

High astigmatism; better visual acuity with glasses

High astigmatism; second aphakic eye of patient happily wearing spectacles

Table 3 Patients taken off the trial (8 cases)

\section{Excessive greasing}

Eye rubbing (deaf patient)

Unstable lens (eye rubbing)

Unstable lens (four lenses in two months)

Iris incarceration followed by increased intraocular tension leading to trabeculectomy

Left the area for India

Left the area and after two episodes of keratitis

Died

Table 4 Keratometry during lens fitting

\begin{tabular}{llc}
\hline & Average \\
Flattest reading at six weeks & $7 \cdot 1-8.5$ & $7 \cdot 76$ \\
Flattest reading at six months & $7 \cdot 1-8.4$ & 7.89 \\
\hline $\begin{array}{l}\text { Average of mean keratometry readings at six weeks } \\
\text { Average of mean keratometry readings at six } \\
\text { months }\end{array}$ & $\mathbf{7 . 6 8}$ \\
\hline
\end{tabular}

Lenses given-20 of $7.8(53 \%)$ and 18 of $8.1(47 \%)$

Table 5 Ocular spherical correction for the series

\begin{tabular}{lll}
\hline Maximum +21.25 & Minimum + 9.25 & Average + 14.04
\end{tabular}

Average soft lens power prescribed $+15 \cdot 12$

Comparison of the ocular spherical correction and power of lens supplied (Table 5) shows the presence (between soft lens and cornea) of a $-1.0 \mathrm{D}$ liquid lens. This was considerably more than is usual in soft lens fitting and indicated the influence of lens rigidity and the semi-scleral fit (the aphakic lens being relatively thick) against the normal peripheral corneal flattening. It also confirmed our clinical impression that our fitting criteria did not result in an especially steep fitting lens.

The keratometric cylinder is shown (Table 6) to have the highest value, and a progressive reduction of this figure is found in the ocular astigmatism (back 
Table 6 Cylindrical correction

\begin{tabular}{|c|c|c|c|}
\hline $\begin{array}{l}\text { Keratometric cylinder } \\
\text { Spectacle cylinder } \\
\text { Converted to ocular } \\
\text { cylinder }\end{array}$ & $\begin{array}{l}\text { Max. } 8 \cdot 62 \\
\text { Max. } 4 \cdot 0 \\
\text { Max. } 5 \cdot 62\end{array}$ & $\begin{array}{l}\text { Min. Nil } \\
\text { Min. Nil } \\
\text { Min. Nil }\end{array}$ & $\begin{array}{c}\text { Average } \\
2 \cdot 60 \\
1 \cdot 70 \\
2 \cdot 0\end{array}$ \\
\hline Average cylindrical spe & overcorrect & (all cases) & $1 \cdot 35$ \\
\hline
\end{tabular}

vertex distance corrected spectacle cylinder) and that required at contact lens overcorrection. Comparing those given with those not given overcorrections we had, as with other soft lenses, an acceptance of less than 2.0 D cylinder without an overcorrection. The average overcorrection cylinder prescribed was $2.43 \mathrm{D}$. The number of overcorrections given was $15(39.47 \%)$, a higher figure than in the previous studies and may account for the good acuity (taking the best visions recorded) compared with spectacle refraction-both average at 6/9.

Technical problems lay in the specifications of lenses. The difficulties of checking soft contact lenses are well known. Therefore without extreme accuracy in production and reproduction by the manufacturers troubles will continue to occur. This problem exists to some extent with all types of highpower soft lenses. However, in this trial powers varied and, worse, cylinders were sometimes present. The troubles often seemed to lie in variations of overall thickness and consequent alteration in 'wrapping' from lens to lens. Nevertheless the represcribing of an overcorrection because a replacement lens was not the same as its predecessor is both expensive and depressing. Which was at fault, the first or the second lens? During the course of the trial this situation improved considerably, some patients achieving $6 / 6$ vision at six months without an overcorrection (Fig. 1, colour plate).

Of the problems that occurred during the followup, assessment is most satisfactorily achieved by examining lens replacement, as no matter whether the problem was corneal or lens orientated the lens was removed and replaced. Thirty-six were replaced (or 1.94 per person per six months including the initial lens), a happier picture than with daily wear (three to four lenses per patient per six months). The vast majority of these were lost, broken, or changed because of deposits-some $75 \%$ (Table 7). Many of those with deposits might have been reclaimed by a cleaning/renovation process, thereby reducing the replacement rate still further to 1.42 per patient per six months.

The other important considerations are the infections. In this series there were four cases in which lenses were changed. Table 8 shows five cases, as the patient with the meibomian cyst had a very mild episode that was treated with antibiotics without lens removal. The first three are the serious ones. The first 'makes sense' in that corneal contact lens intolerance is well known with upper respiratory tract infections, and so one might expect a person using a continuous-wear soft contact lens to be at risk. The second brings up the problem of patients moved beyond our care and the increased susceptibility of those patients who produce deposits. The third is the most worrying, as it would seem to relate to something done to the lens by us, possibly the disturbance of a balanced tear film caused by the lens change. All these patients responded satisfactorily to treatment and are now again wearing their lenses.

Since the trial two patients have developed some degree of peripheral vascularisation and an attempt is being made to overcome this by having the lens made with a flattened peripheral curve in order to achieve more edge lift. Early observations suggest that this will work.

Deposits on these lenses are the greatest drawback to aphakic continuous wear, because once deposits have developed the lens becomes unstable, liable to contamination and infection, often uncomfortable, and certainly difficult to clean. In continuous wear the soft mucous deposits of daily wear have been replaced by discrete masses of rocky appearance that can coalesce to give large conglomerates (Fig. 2 , colour plate). They are usually axial but do not necessarily start off that way, as a single dot once formed will tend to locate in the interpalpebral aperture, obstruct blinking, and develop axially.

The original formation of a deposit certainly relates to poor blinking over these lenses of great

Table 7 Lenses replaced

\begin{tabular}{lrr}
\hline Lost & 5 & $(14 \%)$ \\
Broken & 10 & $(28 \%)$ \\
Deposits & 12 & $(33 \%)$ \\
Radius change & 4 & $(11 \%)$ \\
Power change & 1 & $(3 \%)$ \\
Infection & 4 & $(11 \%)$ \\
\hline Total & $36(100 \%)$ \\
\hline
\end{tabular}

\section{Table 8 Infections: five cases}

1. Corneal ulcer and acute uveitis associated with 'flu' (faint nebula remaining).

2. Severe keratitis while on holiday abroad; patient prone to deposits (faint nebula remaining).

3. Small limbal abscesses with generalised keratitis-3 days-after a lens change because of deposits ( $E$. coli cultured) (faint nebula remaining).

4. Conjunctivitis associated with a meibomian cyst.

5. Conjunctivitis-10 days after a lens change.

All patients again now wearing their lenses. 


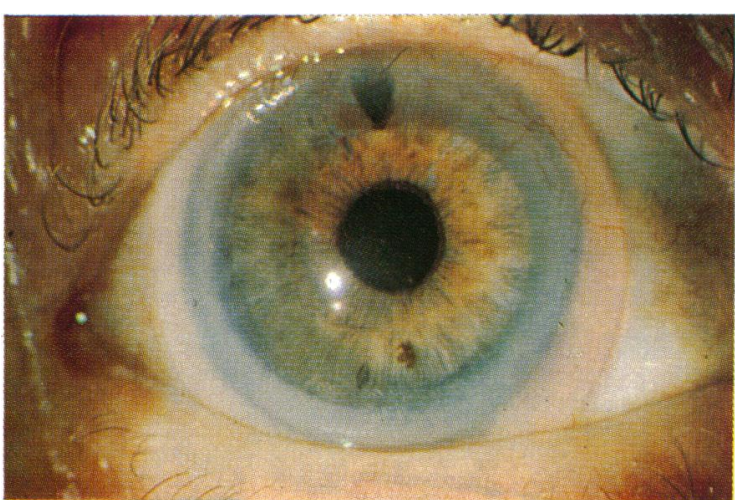

Fig. 1 (Kersley et al.) Aphakic 'soft' contact lens worn continuously for six months achieving a visual acuity of $6 / 6$ without overcorrection.

centre thickness. But other features play a part. The decreased water fraction in the tears of older people further complicates inadequate blinking. Certainly differential drying and temperature gradients across these thick lenses will encourage precipitation, and the fluctuating temperatures produced by lid and lens movement could produce deposition without any other help (Cordrey, 1976). Such precipitations will, of course, adhere to any surface irregularity or deformity (Tripathi and Ruben, 1976) and tear the surface apart (Fig. 3). These deposits have been estimated to contain calcium by autoanalysis of washings obtained from our contaminated lenses (Ganju and Cordrey, 1974; Ruben et al., 1975). In the milky washings from some lenses a high reading was also found for chloride, but the significance of this is unknown.

Two solutions are available for use in removing deposits on Sauflon lenses where the corneal detergent action of methyl cellulose is ineffective. The first is a mixture of a copolymeric dispersant and sodium perberate as an oxidising agent, and it works satisfactorily on mucoprotein deposits, while the second contains the same dispersant but EDTA as the active agent for removing calcium-based deposits. The effectiveness of cleaning can be checked in a before-and-after method by ultraviolet spectography. As both these agents would be very dangerous in the eye, double heat treatment in saline is necessary before lenses are fit to be returned to the eye.

That the selection of patients must be considered very carefully for continuous wear is a factor that has become increasingly more important. It is the patient selection that will alter the statistics of ensuing problems and mark success versus failure

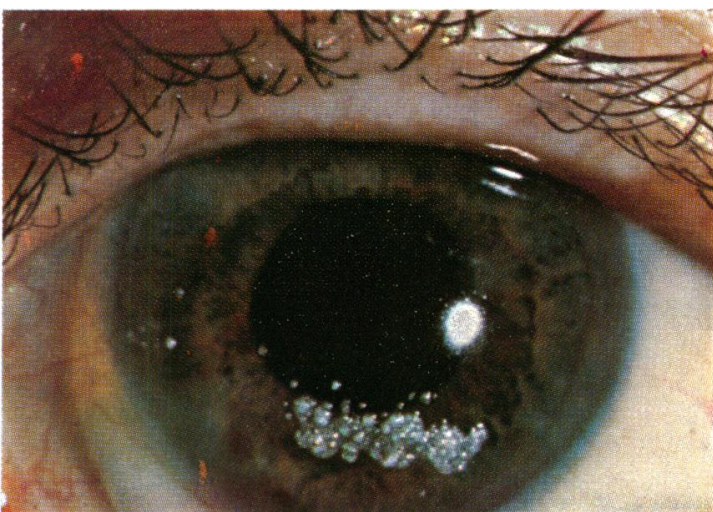

Fig. 2 (Kersley et al.) Aphakic 'soft' contact lens for continuous wear showing the hard deposits that can develop.

from the patient's point of view. The question that has been asked, 'What do you do with the aphakic patient that has to be taken off continuous wear?' has to some extent answered itself. At six weeks patients are still relatively unused to their new vision, and careful consideration of their future and their existing circumstances may result in corneal contact lens fitting or even the prescription of aphakic spectacles. This can be carried out with relatively little disturbance to the patient at this early stage. The points to be considered are: (a) The patient's age: in younger patients a corneal contact lens would be favoured. (b) Their visual requirements; people neither going out a lot nor reading much would possibly be happy with a slightly reduced visual acuity and the absence of handling problems which the continuous wear lens affords. (c) Patients with physical handicaps; these are commoner in the aphakic group, and the patients would be better served by a continuous wear lens. $(d)$ The in-

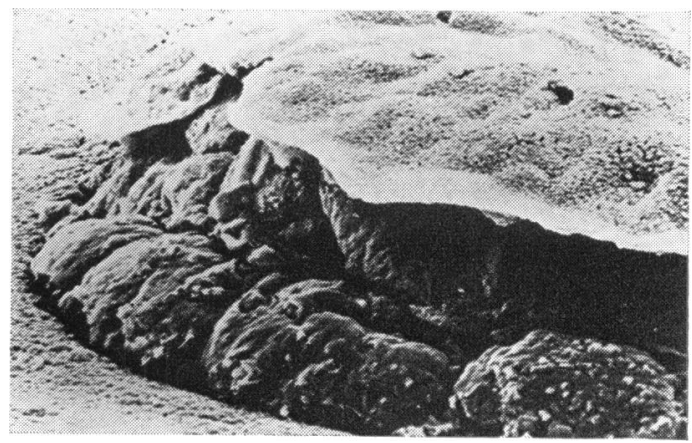

Fig. 3 Electron micrograph to show damage to a soft lens caused by a hard deposit. 
ability of patients to co-operate and attend hospital again for regular check-ups or if problems occur is a factor that may argue against such a lens.

We acknowledge the generous participation of Contact Lenses Manufacturing Ltd in the supply of lenses and cleaning materials.

\section{References}

Cordrey, P. (1976). Analysis of problems encountered in continuous wear soft lenses. Eye, 1, 12.

Ganju, S. N., and Cordrey, P. (1974). U.V. Spectro-photo- metry. A study of hydrophilic lenses with a new cleaning agent. Contact Lens, 15, 8-11.

Kersley, H. J., and Gasson, A. (1975). The aphakic hydrophilic lens in hospital practice. Contacto 19, No. 3, 14-20. Kersley, H. J. (1975). The fitting of soft lenses in aphakia. Optician, 170, 12-18.

Larke, J., and Hirii, N. (1974). Ocular response and soft lens wear: A review. British Journal of Ophthalmic Opticians, 29, 139-134.

Ruben, M., Tripathi, R. C., and Winder, A. F. (1975). Calcium deposition as a cause of spoilation of hydrophilic lenses. British Journal of Ophthalmology, 59, 141.

Tripathi, R. C., and Ruben, M. (1976). Ultrastructure of soft contact lenses. Contact Lens Society Conference, Windermere (in press). 